\title{
Convalescent plasma is of limited clinical benefit in critically ill patients with coronavirus disease-2019: a cohort study
}

\author{
Li Huang ${ }^{1,2 \dagger}$, Che Zhang ${ }^{2,3+}$, Xihui Zhou ${ }^{3}$, Zhou Zhao ${ }^{4}$, Weiping Wang ${ }^{5}$, Weidong Leng ${ }^{2}$, Xiao Su ${ }^{1 *}$ and \\ Qizhou Lian ${ }^{1,6,7^{*}}$ (1)
}

\begin{abstract}
Background: Recently, convalescent plasma (CP) transfusion was employed for severe or critically ill patients with coronavirus disease-2019. However, the benefits of CP for patients with different conditions are still in debate. To contribute clinical evidence of CP on critically ill patients, we analyze the characteristics and outcomes of patients with or without CP transfusion.

Methods: In this cohort study, 14 patients received CP transfusion based on the standard treatments, whereas the other 10 patients received standard treatments as control. Clinical characteristics and outcomes were analyzed. The cumulative survival rate was calculated by Kaplan-Meier survival analysis.

Results: Data analysis was performed on 24 patients (male/female: 15/9) with a median age of 64.0 (44.5-74.5) years. Transient fever was reported in one patient. The cumulative mortality was 21\% (3/14) in patients receiving CP transfusion during a 28-day observation, whereas one dead case $(1 / 10)$ was reported in the control group. No significant difference was detected between groups in 28-day mortality $(P=0.615)$ and radiological alleviation of lung lesions $(P=0.085)$.

Conclusion: In our current study, CP transfusion was clinically safe based on the safety profile; however, the clinical benefit was not significant in critically ill patients with more comorbidities at the late stage of disease during a 28-day observation.
\end{abstract}

Keywords: Convalescent plasma, Coronavirus, Coronavirus disease-2019, Mortality, SARS-CoV-2

\section{Background}

Infection with severe acute respiratory syndrome coronavirus 2 (SARS-CoV-2) has spread worldwide rapidly.

\footnotetext{
*Correspondence: xsu@ips.ac.cn; qzlian@hku.hk

${ }^{\dagger}$ Li Huang and Che Zhang have contributed equally to this work and share first authorship

${ }^{1}$ The Joint Center for Infection and Immunity, Guangzhou Institute of Pediatrics, Guangzhou Women and Children's Medical Center, Guangzhou 510623, China; Institute Pasteur of Shanghai, Chinese Academy of Science, Shanghai 200031, China

${ }^{7}$ HKUMed Laboratory of Cellular Therapeutics, The University of Hong Kong, Hong Kong, China

Full list of author information is available at the end of the article
}

SARS-CoV-2 has been identified as the cause of coronavirus disease-2019 (COVID-19) that is considered to be a diverse clade derived from severe acute respiratory syndrome coronavirus (SARS-CoV) and Middle East respiratory syndrome coronavirus (MERS-CoV) [1]. The current treatment strategy mainly relies on antiviral therapy and supportive treatment [2]. Immunotherapies have been applied based on therapeutic plasma exchange [3], mesenchymal stem cell transfusion [4-6], and convalescent plasma (CP) transfusion [7]. Preliminary clinical benefits of $\mathrm{CP}$ transfusion have been shown in specific patient populations, including severe [8] or critically ill patients original author(s) and the source, provide a link to the Creative Commons licence, and indicate if changes were made. The images or other third party material in this article are included in the article's Creative Commons licence, unless indicated otherwise in a credit line to the material. If material is not included in the article's Creative Commons licence and your intended use is not permitted by statutory regulation or exceeds the permitted use, you will need to obtain permission directly from the copyright holder. To view a copy of this licence, visit http://creativecommons.org/licenses/by/4.0/. The Creative Commons Public Domain Dedication waiver (http://creativeco mmons.org/publicdomain/zero/1.0/) applies to the data made available in this article, unless otherwise stated in a credit line to the data. 
with no more than two comorbidities in some case series [9-12] and case-controlled studies[13]; however, no significant difference was detected in patients with or without CP transfusion based on the 28-day mortality in a randomized clinical trial [14]. The benefits of CP transfusion for patients with different conditions are still in debate. In this cohort study, we present the clinical characteristics and outcomes of 24 critically ill patients with COVID-19, who received treatments with or without CP transfusion.

\section{Methods}

\section{Study setting, sample size, subjects, and ethical approval}

This cohort study set out to evaluate the safety and potential benefit of CP transfusion in critically ill patients with COVID-19. The sample size was calculated using PASS software (version 15.0, NCSS, LLC. Kaysville, Utah, USA). An estimated sample size of 56 (28 patients in a group) would provide $80 \%$ power ( $\alpha=0.05$, two-tail) for an analysis of 28-day mortality, given estimated mortality as $25 \%$ in the CP exposed group [15] and $61.5 \%$ in the unexposed group [16]. Patients were recruited from four hospitals in West China. Patients in the CP group received the standard treatment with $\mathrm{CP}$ according to the criteria defined in the recommendations of the National Health Commission of the People's Republic of China (NHC) [17], who were matched to the control group. The study was reported following the STROBE reporting guideline.

Patients who fulfilled the following criteria were included: (1) diagnosed with laboratory-confirmed COVID-19 according to the World Health Organization (WHO) guideline [18] and the recommendations of the NHC [19]; (2) identified as critically ill according to the recommendations of the NHC [19] (Additional file 1: Table S1); (3) written informed consent was obtained; (4) age $\geq 18$ years. Patients who received other investigational treatments were excluded for analysis, including but not limited to mesenchymal stem cell transfusion.

Ethical approval was obtained from the institutional review board of the Affiliated Taihe Hospital of Hubei University of Medicine (Ethical Approval 2020KY04, 2021KS033). Written informed consent was obtained from the patients and/or guardians before data collection.

\section{Data collection and assessments}

Clinical data were retrieved from medical charts by the study team. Data were cross-checked by two researchers for quality control. SARS-CoV-2 RNA was detected by real-time reverse transcription-polymerase chain reaction according to the recommendations of the NHC [20]. A sputum sample or nasopharyngeal/throat swab was taken for testing. Hematological, serum biochemical, and coagulation tests were performed. Chest computed tomography $(\mathrm{CT})$ scans were conducted. In patients with limited mobility, digital radiography was performed at the bedside. Images were stored in picture archiving and communication systems, and reviewed by two experienced radiologists independently. Dissenting opinions between the radiologists were determined by consensus.

\section{Standard treatments}

All patients received antiviral therapy in the form of interferon- $\alpha$ nebulization and arbidol. Antibiotic therapy was prescribed for patients with bacterial pneumonia. Glucocorticoids and mechanical ventilation were initiated if indicated.

\section{Preparation and transfusion of $\mathrm{CP}$}

Donor recruitment, CP collection, quality control, storage, and transportation were performed by Sino Pharm and Wuhan Blood Bank, the specific organizations designated by the NHC in Wuhan. Donors were recruited according to the criteria defined in the recommendations of the NHC [17] and WHO [21] as follows: (1) previously diagnosed with laboratory-confirmed COVID-19; (2) complete resolusion of symptoms at least for 3 weeks before donation; (3) met the criteria for discharge from hospital and quarantine, including negative SARS-CoV-2 RNA test; (4) positive serological test for SARS-CoV-2 antibodies; (5) aged between 18 and 55 years; (6) eligible in the physical exams according to the requirement of national standard operating procedures of blood collection [22], including vital signs and body weight (body weight $\geq 50 \mathrm{~kg}$ for male or $\geq 45 \mathrm{~kg}$ for female); (7) obtained the information of $\mathrm{ABO}$ blood type and eligible in the pretest of blood sample for hemoglobin and alanine aminotransferase; (8) negative in pregnancy test for female donors; (9) negative in HNA and HLA antibody tests for donors with history of gestation or blood transfusion; (10) without a history of transfusion-transmitted infectious disease, and negative for pathogens of transfusion transmitted diseases, including but not limited to hepatitis $B$ virus, hepatitis $C$ virus, human immunodeficiency virus, and syphilis; (11) suitable for donating according to the judgment of physician; (12) written informed consent was obtained.

The plasma from donors was collected and processed by trained staff with the nurse or medical certificates in Sino Pharm and Wuhan Blood Bank, which were certified for routine collection and preparation of blood and plasma in accordance with NHC guidelines [22, 23]. The convalescent plasma was prepared from apheresis collection. Briefly, the plasma was collected in an enclosed system with a blood cell separator, the procedure of which included blood collecting, anticoagulant agent inpouring, 
blood cell separating, plasma collecting, and blood cells transfusion back to the donor. Essential information was provided on the package of plasma with a unique barcode for source tracking. The plasma was kept at $2-6{ }^{\circ} \mathrm{C}$ within $48 \mathrm{~h}$, or freeze at $-20{ }^{\circ} \mathrm{C}$ for long-term storage. The plasma samples for release testing were divided from the donated plasma. The quality of CP was checked to meet the following criteria before releasing to clinical centers, including negative for SARS-CoV-2 RNA, hepatitis B virus, hepatitis $C$ virus, human immunodeficiency virus, and syphilis; positive for SARS-CoV-2 specific IgG antibodies in 160 times dilution of serum samples, or positive for SARS-CoV-2 specific total antibodies in 320 times dilution by enzyme-linked immunosorbent assay or chemiluminescence [17]. CP was released to designated treatment centers for clinical use with the approval of the Hubei Provincial Centre for Disease Control and Prevention. CP transfusion was completed within $24 \mathrm{~h}$ of receipt following the procedure in the recommendation of NHC [17]. Briefly, patient status was reviewed by physicians to check if any contraindication existed before transfusion, including allergic history to plasma or sodium citrate, or other situation not allowing transfusion. The dosage was calculated according to the bodyweight of the patient 3-5 ml/kg with an upper limitation of $250 \mathrm{ml}$ per dose. Patients were planned to allocate into two subgroups according to their acute physiology and chronic health evaluation II (APACHE II) scores in a 1:1 ratio to receive a low or high dosage of $\mathrm{CP}$ from donors with matched $\mathrm{ABO}$ blood types. The patients with less than 10 scores in the evaluation would receive more than $300 \mathrm{ml}$ $(<600 \mathrm{ml}) \mathrm{CP}$ in total volume, while the patients with 10 or more scores in APACHE II would receive $600 \mathrm{ml}$ or more $(<900 \mathrm{ml})$ in total volume. Initial transfusion was conducted in the intensive care unit under close monitoring. The duration of transfusion was over one hour for risk management. Thereafter, transfusion was finished in 40-45 min in patients with good tolerance. The adverse event that occurred during or within $72 \mathrm{~h}$ of transfusion was recorded.

\section{Outcomes}

The primary end-point of this study was the mortality of patients in a 28-day observational period. The secondary end-points included radiological alleviation of lung lesions in a 7-day and 28-day period, which was assessed by a CT scoring system for lung lesions [24] (Additional file 1: Table S2). The total scores were calculated as the sum of the scores for five lung lobes. Radiological alleviation was considered when the total scores reduced at least two points or changed to zero. The alleviation rate was analyzed between groups. The cut-off date for survival analysis was 28 days after initial intervention in ICU, except that the patient was discharged or dead before the cut-off date. Patients were discharged if they fulfilled the criteria in recommendations of NHC [19] as follows: (1) fever had recovered for 3 days at least; (2) upper respiratory symptoms were alleviated significantly; (3) the exudative lesion was alleviated remarkably based on radiological images; (4) SARS-CoV-2 nucleic acid was negative in two consecutive tests with an interval of $24 \mathrm{~h}$.

\section{Statistical analysis}

Statistical analysis was performed using SPSS software (version 20.0, International Business Machines Corporation, Armonk, NY, USA). Continuous variables are presented as the median (interquartile range) or mean \pm stand error. Categorical variables are presented as number and frequency rates. No imputation was used for missing data. A two-tailed T-test was used to compare continuous variables between groups. The Chisquare test or Fisher's exact test was used to compare categorical variables between groups. The cumulative survival rate was calculated by Kaplan-Meier (K-M) survival analysis. The log-rank test was performed in conjunction with $\mathrm{K}-\mathrm{M}$ analysis. Statistical significance was considered if $P<0.05$.

\section{Results}

\section{Patient characteristics}

From January 29 to March 16, 2020, 26 critically ill patients with confirmed SARS-CoV-2 infection were admitted to the hospitals, two of whom were excluded because they had undergone mesenchymal stem cell transplantation (Fig. 1). The recruitment was terminated by the end of March due to the challenge of a limited patient population along with the containment of SARSCoV-2 infection in West China. Therefore, 24 patients (male/female; 15/9) were enrolled totally with a median age of 64.0 (44.5-74.5) years. The final follow-up visit was completed by April 15, 2020. Notably, all patients had comorbidities, numbering three or more in $12(50 \%)$ patients (Table 1). No significant difference was detected in patient characteristics between groups. A notable decrease of albumin and an increase of high-sensitivity C-reactive protein were detected in patients in both groups. No significant difference was detected in the proportion of abnormal parameters between groups (Additional file 1: Table S3).

\section{Treatments}

All patients received interferon- $\alpha$ nebulization at a dose of 2.5 million $\mathrm{IU} / \mathrm{ml}$ twice daily and arbidol at $200 \mathrm{mg} /$ dose three times a day according to the recommendation of the NHC [19]. Short-term systemic methylprednisolone was administered to all patients within 3-5 days at 


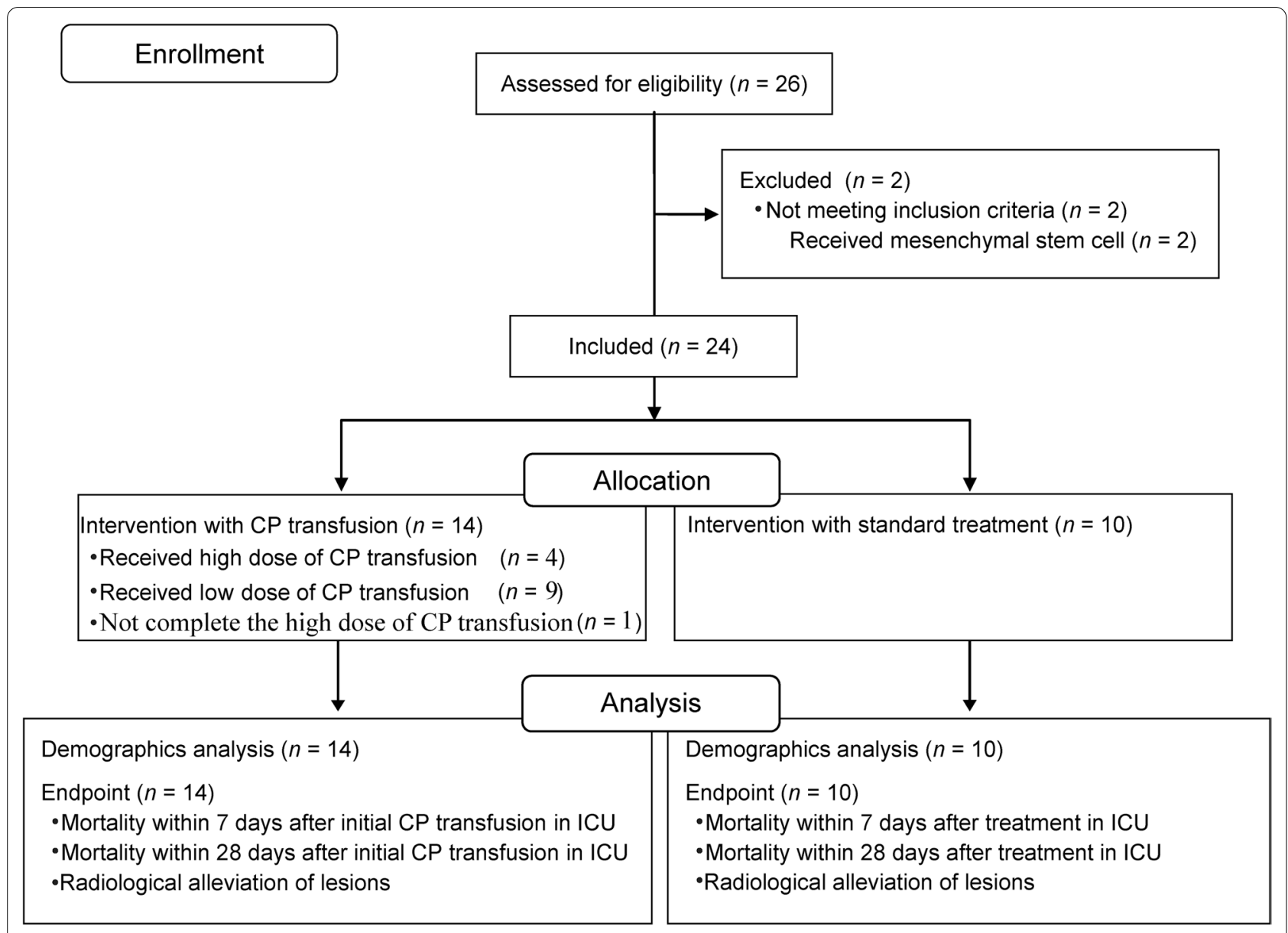

Fig. 1 Clinical process and data analysis. Twenty-four patients were included in the study

a daily dosage of $1-2 \mathrm{mg} / \mathrm{kg}$. Cefoperazone sodium was given to 12 patients $(50 \%)$ with complicating bacterial pneumonia at a dose of $40 \mathrm{mg} / \mathrm{ml}$ twice a day. Caspofungin was administered at a dose of $0.27 \mathrm{mg} / \mathrm{ml}$ once a day to seven patients (29\%) with fungal infections in the respiratory tract. Mechanical ventilation was required by 12 patients $(50 \%)$ and extracorporeal membrane oxygenation by one patient. No significant difference was detected between groups in standard treatments. Based on standard treatment, 14 patients received CP transfusion (Fig. 2A) following the recommendations of the NHC $[19,20]$. The initial transfusion of CP was conducted at $20.7( \pm 3.6)$ days after hospital admission (Table 2). Finally, five patients were allocated in a high dosage group to receive at least $600 \mathrm{ml}$ of $\mathrm{CP}$ in total volume, of whom one patient (Pt. No. 1) was dead before receiving the second dosage due to aggressive progression of the disease. Besides, nine patients received low doses of CP (less than $600 \mathrm{ml}$ ), of whom four patients were transmitted from ICU to ordinary wards for relief of fatal symptoms after the first dose of CP (Pt. No. 5, 7, 10, and 11), and did not receive the second doses in accordance with the instruction of NHC [17] (Additional file 1: Table S4).

\section{Outcomes}

A transient fever $\left(37.4{ }^{\circ} \mathrm{C}\right)$ was observed in a patient within $24 \mathrm{~h}$ of the first $\mathrm{CP}$ transfusion but had resolved by the following day. The cumulative mortality was $21 \%$ (three dead cases) in patients who received CP transfusion during a 28-day observational period, whereas one dead case was reported in patients without $\mathrm{CP}$ transfusion. However, no significant difference was detected in 28-day mortality (Table 2), and cumulative survival analysis between groups (Fig. 2B, Log-rank $P=0.321$ ). Further analysis of the clinical course was performed in dead cases with $\mathrm{CP}$ transfusion to identify the underlying reason for poor outcomes. One patient died of multiple organ dysfunction syndromes from extensive disease progression. The other two patients died from 
Table 1 Clinical characteristics of patients $(n=24)$

\begin{tabular}{|c|c|c|c|}
\hline Clinical characteristics & Received CP $(n=14)$ & Without CP $(n=10)$ & $P$ value \\
\hline \multicolumn{4}{|l|}{ Demographics } \\
\hline \multicolumn{4}{|l|}{ Gender } \\
\hline Male, $n(\%)$ & $8(57)$ & $7(70)$ & 0.678 \\
\hline Female, $n(\%)$ & $6(43)$ & $3(30)$ & 0.678 \\
\hline Age, mean $\pm S E, y$ & $64.6 \pm 4.3$ & $55.6 \pm 4.8$ & 0.177 \\
\hline$<40$ years, $n(\%)$ & $1(7)$ & $1(10)$ & 1 \\
\hline $40-60$ years, $n(\%)$ & $3(21)$ & $5(50)$ & 0.204 \\
\hline$>60$ years, $n(\%)$ & $10(71)$ & $4(40)$ & 0.211 \\
\hline $\mathrm{APACHE} \| \mathrm{score}$, mean $\pm \mathrm{SE}$ & $12.2 \pm 1.6$ & $9.0 \pm 0.8$ & 0.121 \\
\hline With three or more comorbidities, $n$ (\%) & $8(57)$ & $4(40)$ & 0.680 \\
\hline \multicolumn{4}{|l|}{ Main comorbidities } \\
\hline Chronic obstructive pulmonary disease, $n(\%)$ & $6(43)$ & $1(10)$ & 0.171 \\
\hline Diabetes mellitus, $n(\%)$ & $4(29)$ & $1(10)$ & 0.358 \\
\hline Hypertension, $n$ (\%) & $4(29)$ & $1(10)$ & 0.358 \\
\hline Coronary heart disease, $n(\%)$ & $4(29)$ & 0 & 0.114 \\
\hline \multicolumn{4}{|l|}{ Main complication } \\
\hline Bacterial pneumonia, $n(\%)$ & $9(64)$ & $3(30)$ & 0.213 \\
\hline Hypoalbuminous edema, $n$ (\%) & $8(57)$ & $3(30)$ & 0.240 \\
\hline Respiratory fungal infection, $n(\%)$ & $6(43)$ & $1(10)$ & 0.171 \\
\hline Gastrointestinal hemorrhage, $n(\%)$ & $2(14)$ & 0 & 0.493 \\
\hline
\end{tabular}

APACHE acute physiology and chronic health evaluation, CP convalescent plasma, SE stand error

deterioration of their comorbidities (Additional file 1: Figure S1), including a cerebral hernia and a relapse of pulmonary tuberculosis respectively. All these events were judged not relative to $\mathrm{CP}$ transfusion.

SARS-CoV-2 RNA became negative for all patients after CP transfusion. Radiological alleviation of lung lesions was detected within 28 days after initial intervention in 12 patients who received CP transfusion and five patients without transfusion, although the difference was not significant statistically $(P=0.085$, Table 2$)$. Notable improvements were shown in patient No. 4 after $\mathrm{CP}$ transfusion (Fig. 3E), who had no severe pulmonary comorbidities and shown better performance in serologic assays for prognosis indicators during the treatment (Additional file 1: Figure S2). No improvement was detected in digital radiography for patient No. 13 (Fig. 3F) who had severe pulmonary comorbidities and poor prognosis according to the serologic assays (Additional file 1: Figure S2).

\section{Discussion}

Currently, the therapeutic strategy for patients with COVID-19 mainly relies on antiviral therapy and supportive treatment. Although some treatments with neutralizing antibodies have shown benefits in preclinical studies [25] and outpatients with COVID-19 [26], the therapeutic potential in critically ill patients with
COVID-19 is still under research. As a substitute of monoclonal antibodies for passive immunity, transfusion of $\mathrm{CP}$ has been suggested as an option for critically ill patients by the WHO [27] and the NHC [17] based on its empirical utilization in SARS virus infections [28]. Nevertheless, it is very challenging to rescue such critically ill patients who usually died from ARDS or multiple organ failure caused by SARS-CoV-2-provoked destructive immune responses and inflammatory complications. In agreement with previous reports [29], we found that the virus-activated CRS was unlikely to be reversed by CP transfusion, although SARS-CoV-2 RNA did become negative in all patients and alleviation of lung lesions was observed in some patients after CP treatments. Unlike other reports, $12(50 \%)$ patients in our study had three or more comorbidities. Clinical data analysis was performed between patients with or without CP transfusion to evaluate the safety and therapeutic potential of $\mathrm{CP}$ in our patient population.

Early safety data were favorable for $\mathrm{CP}$ transfusion in a study with 20,000 in-patients with severe or life-threatening COVID-19. Serious adverse events were reported with a low incidence, including transfusion reactions $(<1 \%)$, thromboembolic or thrombotic events $(<1 \%)$, and cardiac events (around 3\%). And the vast majority of the thromboembolic or thrombotic events and cardiac events were judged to be unrelated to $\mathrm{CP}$ transfusion 


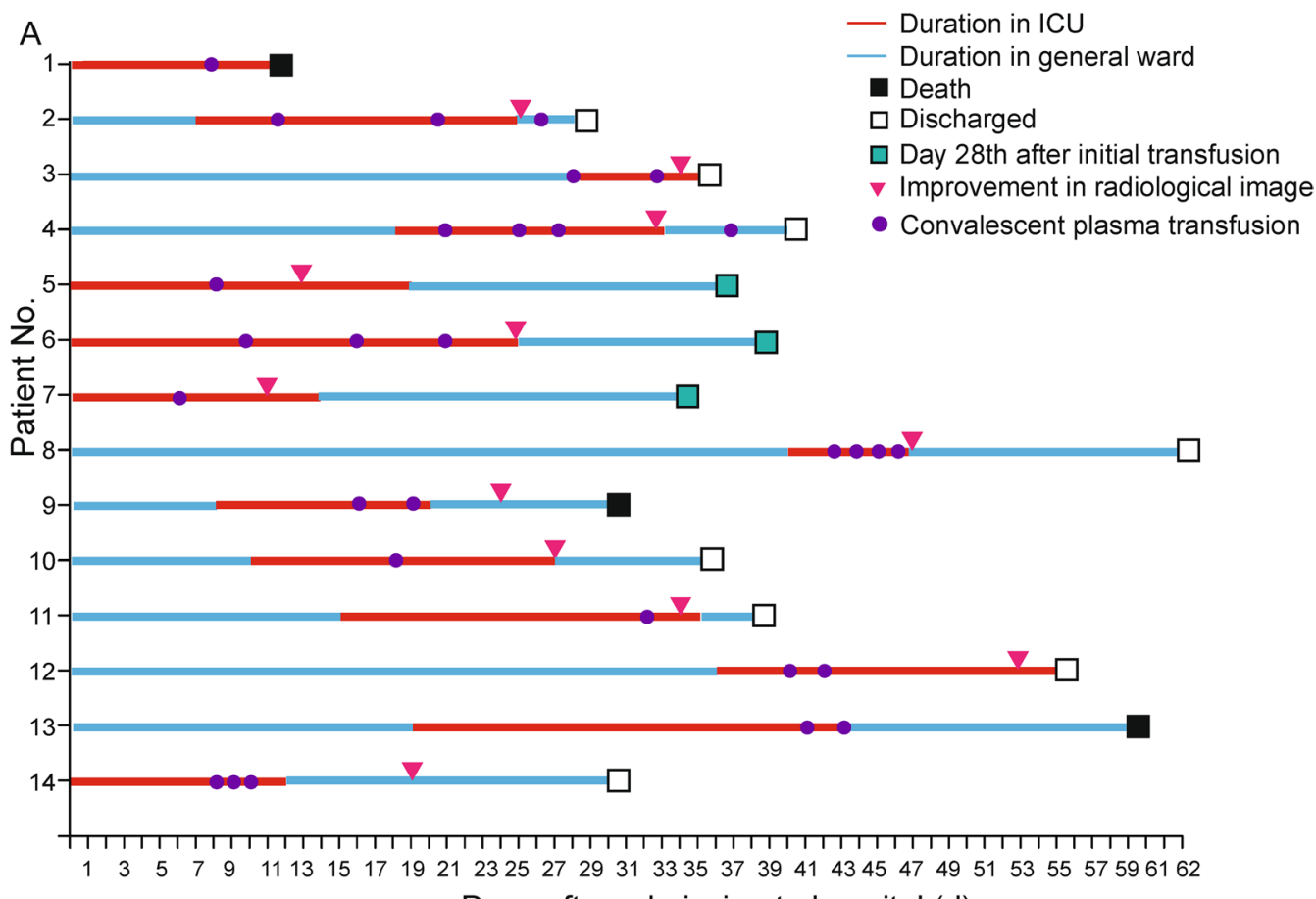

Days after admission to hospital (d)

$\mathrm{B}$

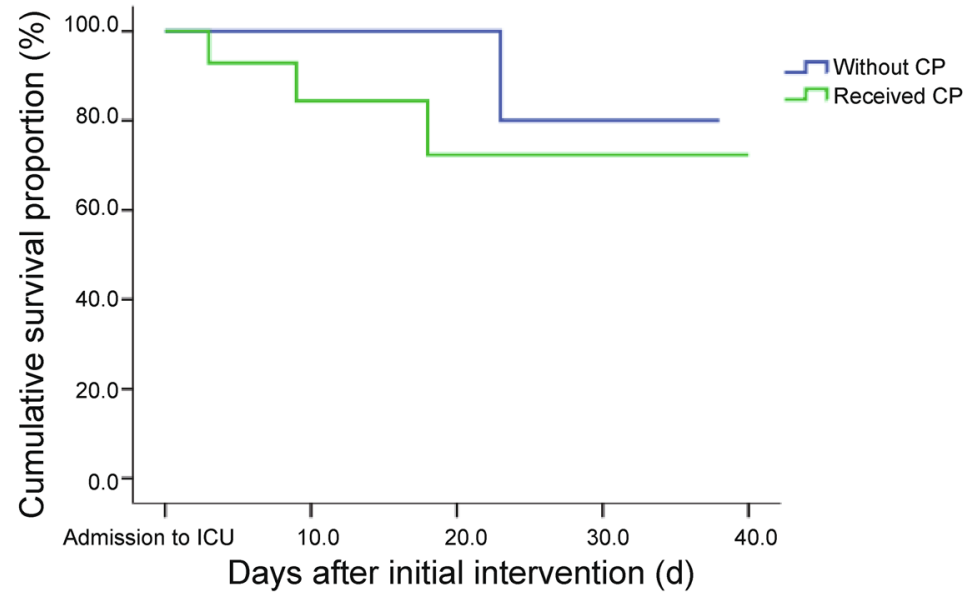

Fig. 2 Clinical course of patients in CP transfusion group and cumulative survival analysis. (A) Fourteen patients received the initial convalescent plasma transfusion in the intensive care unit. Three patients died within 28 days after the initial transfusion. (B) Cumulative survival incidence was calculated by K-M survival analysis. No significant difference was detected between the two groups with or without CP transfusion (Log-rank $P=0.321)$

[30]. In our study, no serious adverse event was reported. One case of transient fever was reported linked to $\mathrm{CP}$ transfusion. It was indicated that $\mathrm{CP}$ transfusion would not increase the risk of mortality since no significant difference was detected in the mortality of patients with or without CP transfusion.

The therapeutic efficacy of CP transfusion could be affected by initial transfusion time basing on a study of SARS, which suggested an initial transfusion within
14 days of symptom onset for better outcomes [31]. It was indicated in some case series that severe and critically ill patients could benefit from $\mathrm{CP}$ transfusion if given as early as 2 days [32, 33], 3 days [34, 35], $4.5( \pm 1.0)$ days [36], and $13.8( \pm 6.5)$ days after hospitalization [37]. Neutralizing antibodies and the antiinflammatory effect of $\mathrm{CP}$ were indicated to contribute to an attenuation of hypoxia after CP transfusion [38]. In a previous study with a single dose transfusion, the 
Table 2 Treatments and outcomes of patients $(n=24)$

\begin{tabular}{|c|c|c|c|}
\hline Clinical course & Received CP $(n=14)$ & Without CP $(n=10)$ & $P$ value \\
\hline \multicolumn{4}{|l|}{ Treatments } \\
\hline Antiviral, $n(\%)$ & $14(100)$ & $10(100)$ & 1 \\
\hline Glucocorticoids, n (\%) & $14(100)$ & $10(100)$ & 1 \\
\hline Antibiotics, $n(\%)$ & $9(64)$ & $3(30)$ & 0.213 \\
\hline Antifungal, $n(\%)$ & $6(43)$ & $1(10)$ & 0.171 \\
\hline Low-flow nasal cannula, $n$ (\%) & $4(29)$ & $7(70)$ & 0.095 \\
\hline Mechanical ventilation, $n(\%)$ & $9(64)$ & $3(30)$ & 0.214 \\
\hline Extracorporeal membrane oxygenation, $n(\%)$ & $1(7)$ & 0 & 1 \\
\hline \multicolumn{4}{|l|}{$\mathrm{CP}$ transfusion } \\
\hline Low dose, $n(\%)$ & $9(69)$ & - & - \\
\hline High dose, $n(\%)$ & $4^{\mathrm{a}}(31)$ & - & - \\
\hline Initial transfusion time, mean $\pm S E, \mathrm{~d}^{\mathrm{b}}$ & $20.7 \pm 3.6$ & - & - \\
\hline \multicolumn{4}{|l|}{ Mortality } \\
\hline 7-day mortality, $n(\%)^{c}$ & $1(7)$ & 0 & 1 \\
\hline 28-day mortality, $n(\%)^{\mathrm{d}}$ & $3(21)$ & $1(10)$ & 0.615 \\
\hline \multicolumn{4}{|l|}{ Radiological alleviation of lesions } \\
\hline 7-day alleviation proportion, $n(\%)^{c}$ & $5(36)$ & $2(20)$ & 0.653 \\
\hline 28-day alleviation proportion, $n(\%)^{d}$ & $12(86)$ & $5(50)$ & 0.085 \\
\hline
\end{tabular}

$C P$ convalescent plasma, SE stand error

a Patient No. 1 was excluded from the analysis since she could not finish the high-dose therapeutic scheme due to the death of aggressive progression before the second dose

${ }^{\mathrm{b}}$ Days from hospital admission to initial transfusion of $\mathrm{CP}$

${ }^{c}$ Within 7 days after initial CP transfusion for patients received $\mathrm{CP}$ or treatment without $\mathrm{CP}$ in ICU

${ }^{d}$ Within 28 days after initial CP transfusion for patients received CP or treatment without CP in ICU

28-day mortality of critically ill patients was reported to be $28 \%(8 / 28)$ in the CP treatment group, and $35 \%$ $(10 / 28)$ in the control group; however, no significant difference was detected between groups [14]. In a randomized, placebo-control trial, 228 patients with COVID-19 severe pneumonia received CP transfusion at 8 (5-10) days after symptoms onset. No significant differences were observed in clinical status or 30-day mortality between patients treated with (mortality $10.9 \%$ ) or without (mortality $11.4 \%$ ) CP [39]. With regards to the patients with moderate COVID-19, it was indicated that CP wasn't associated with a reduction in progression to severe COVID-19 or all-cause mortality at 28 days after transfusion at $8(6-11)$ days upon symptoms onset in an open-label, randomized, controlled trial [40]. It was also indicated in a systematic analysis that the effectiveness of $\mathrm{CP}$ treatment was uncertain on all-cause mortality at up to day 28 in patients with moderate and severe COVID-19 [41]. In our study with a repeated transfusion strategy for critically ill patients with more comorbidities, the 28-day mortality was $21 \%(3 / 14)$ in patients with CP transfusion and $10 \%(1 / 10)$ in the control group without $\mathrm{CP}$ treatment. In accordance with previous studies, no significant difference was detected between groups, although the initial transfusion was conducted earlier $(20.7 \pm 3.6$ days) than that in a previous study ( 26 days, IQR 20-36 days) [14].

Critically ill patients could benefit from CP transfusion to achieve pneumonia alleviation in studies with four cases [10] and six cases [11]. However, clinical improvement was not significant between patients with or without CP transfusion within a 28 -day observation [14]. In our study, alleviation of lung lesions was evident in $86 \%(12 / 14)$ patients who received CP transfusion; however, the difference could not reach a statistically significant level $(P=0.085)$ between groups with or without $\mathrm{CP}$ transfusion. Further study with a larger population was needed to identify the potential of $\mathrm{CP}$ on pneumonia alleviation for critically ill patients with COVID-19.

Cytokine release syndrome (CRS) is common in patients with COVID-19 [42] and is believed to be the major cause of morbidity in patients with SARS-CoV and MERS-CoV infection [43]. Recent clinical evidence suggests that the mortality of patients with COVID-19 may be attributed to virus-activated CRS [44]. Increasing levels of interleukin-6, CRP, and other inflammatory 


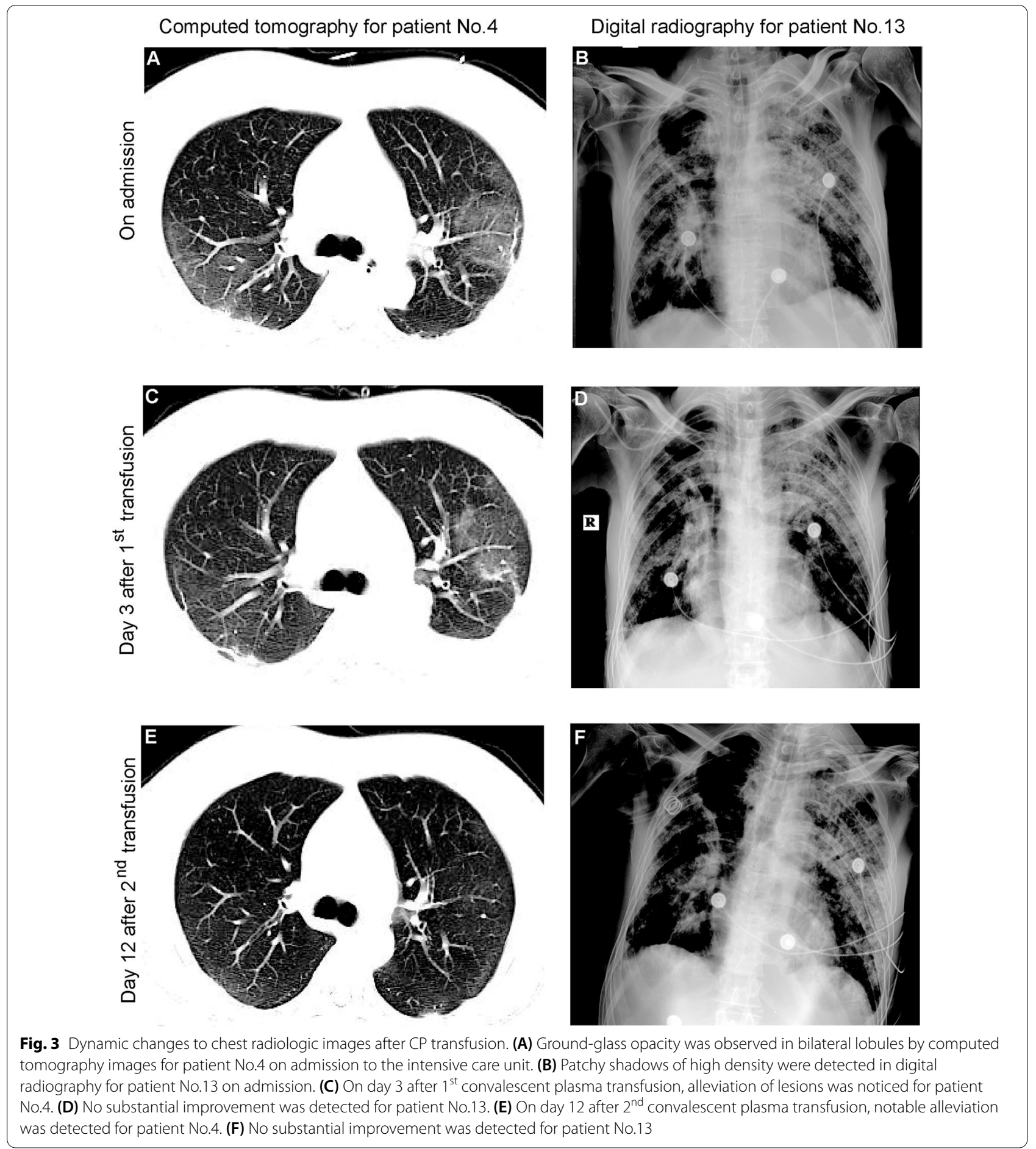

cytokines are reported hallmarks of severe MERS-CoV infection [45] and were evident in almost all patients in our study. It is noteworthy that death in our study was not directly related to the progression of pneumonia, but the deterioration of comorbidities. We believe virus-activated CRS may contribute to the deterioration of comorbidities and lead to a poor clinical outcome. According to the laboratory findings in our study, no significant relevance was observed between the serologic assays of several prognosis indicators and $\mathrm{CP}$ treatments, including hs-CRP (Additional file 1: Figure S2). The virus-activated CRS was unlikely to be 
reversed by $\mathrm{CP}$ transfusion, although SARS-CoV-2 RNA did become negative in all patients and radiological alleviation of lung lesions was observed in some patients after $\mathrm{CP}$ treatments.

\section{Conclusions}

Our findings support the safety of $\mathrm{CP}$ transfusion for clinical use. The clinical benefit of convalescent plasma transfusion was not statistically significant in our patient population basing on the data of 28-day mortality, though radiological alleviation of lung lesions was observed in some patients after CP treatments. Although the neutralizing antibody titer level of the CP was controlled by the designated organizations according to the instruction from the NHC, the specific titer for each dose could not be tested due to the limitation of the local laboratory in the early stage of the pandemic. Besides, the interpretation of our findings is limited by the heterogeneous characteristics of patients with different comorbidities and complications at baseline, which failed to adjust due to the small sample size. Also, CP transfusion could not be conducted in an early stage due to diverse patient referrals in different cases and only severe or critically ill patients were recommended for CP treatment according to the instruction of NHC [17]. Further trials with rigorous designs are needed to determine the optimum subject population and therapeutic time window for better clinical benefits.

\begin{abstract}
Abbreviations
COVID-19: Coronavirus disease-2019; CP: Convalescent plasma; CRS: Cytokine release syndrome; CT: Computed tomography; ICU: Intensive care unit; IQR: Interquartile range; MERS-CoV: Middle East respiratory syndrome coronavirus; NHC: National Health Commission of the People's Republic of China; SARSCoV: Severe acute respiratory syndrome coronavirus; SARS-CoV-2: Severe acute respiratory syndrome coronavirus 2; WHO: World Health Organization.
\end{abstract}

\section{Supplementary Information}

The online version contains supplementary material available at https://doi. org/10.1186/s12967-021-03028-5.

Additional file 1: Table S1. Clinical type of COVID-19. Table S2. CT scoring system for lung lesions. Table S3. Laboratory findings of patients on admission to the intensive care unit $(n=24)$. Table S4. Individual treatment with convalescent plasma transfusion $(n=14)$. Figure S1. Comorbidities and complications of patients received convalescent plasma transfusion $(n=14)$. Figure $\mathbf{S} 2$. Serologic assays for patients received convalescent plasma transfusion $(n=14)$.

\section{Acknowledgements}

We acknowledge the hospital staff for their assistance in data collection. We thank all the patients for donating their data for analysis.

\section{Authors' contributions}

LH and CZ conceptualized the manuscript and took responsibility for data analysis and interpretation with full access to all of the data in the study. XZ, ZZ, WW, and WL contributed to resource, investigation, and data collection. XZ and QL contributed to funding acquisition. LH drafted the manuscript. XS and QL contributed to the review and editing of the manuscript, supervising the work, and correspondence. All authors agree to be accountable for all aspects of the work to appropriately address questions relative to the accuracy or integrity of the work. All authors read and approved the final manuscript.

Funding

This study was in part supported by the National Natural Science Grant of China (Grant No. 31571407); Special Fund to Fight COVID-19 in Guangzhou Women and Children's Medical Center (Grant No. 5001-4001010); and Grant for Clinical Research in Xi'an Jiaotong University (Grant No. XJTU1AF-CRF-2016-023)

\section{Availability of data and materials}

All data generated or analyzed during this study are included in this published article and its Additional file 1.

\section{Declarations}

Ethics approval and consent to participate

Ethical approval was available from the institutional review board (IRB) of the Affiliated Taihe Hospital of Hubei University of Medicine (Ethical Approval $2020 \mathrm{KY} 04,2021 \mathrm{KS} 033)$. Written informed consent was obtained from the patient and/or guardians before data collection.

\section{Consent for publication}

Written informed consent was obtained from the patient and/or guardians before data collection.

\section{Competing interests}

The authors declare that they have no competing interests.

\section{Author details}

${ }^{1}$ The Joint Center for Infection and Immunity, Guangzhou Institute of Pediatrics, Guangzhou Women and Children's Medical Center, Guangzhou 510623, China; Institute Pasteur of Shanghai, Chinese Academy of Science, Shanghai 200031 , China. ${ }^{2}$ Clinical Research Centre, Taihe Hospital, Hubei University of Medicine, Shiyan, China. ${ }^{3}$ Intensive Care Unit, The First Affiliated Hospital of Xi'an Jiaotong University, Xi'an, China. Intensive Care Unit, Shiyan People Hospital, Shiyan, China. ${ }^{5}$ Department of Respiratory Medicine, Shiyan Xiyuan Hospital, Shiyan, China. ${ }^{6}$ Department of Medicine, LKS Faculty of Medicine, The University of Hong Kong, Hong Kong, China. ${ }^{7}$ HKUMed Laboratory of Cellular Therapeutics, The University of Hong Kong, Hong Kong, China.

Received: 17 May 2021 Accepted: 6 August 2021

Published online: 26 August 2021

\section{References}

1. Zhu N, Zhang D, Wang W, Li X, Yang B, Song J, et al. A novel coronavirus from patients with pneumonia in China, 2019. N Engl J Med. 2020;382(8):727-33.

2. Sanders JM, Monogue ML, Jodlowski TZ, Cutrell JB. Pharmacologic treatments for coronavirus disease 2019 (COVID-19): a review. JAMA. 2020;323(18):1824-36.

3. Zhang L, Zhai H, Ma S, Chen J, Gao Y. Efficacy of therapeutic plasma exchange in severe COVID-19 patients. Br J Haematol. 2020:190(4):e181-3.

4. Atluri S, Manchikanti L, Hirsch JA. Expanded umbilical cord mesenchymal stem cells (UC-MSCs) as a therapeutic strategy in managing critically ill COVID-19 patients the case for compassionate use. Pain Physician. 2020;23(2):e71-83.

5. Leng Z, Zhu R, Hou W, Feng Y, Yang Y, Han Q, et al. Transplantation of ACE2(-) mesenchymal stem cells improves the outcome of patients with COVID-19 pneumonia. Aging Dis. 2020;11(2):216-28.

6. Feng $Y$, Huang J, Wu J, Xu Y, Chen B, Jiang $L$, et al. Safety and feasibility of umbilical cord mesenchymal stem cells in patients with COVID-19 pneumonia: a pilot study. Cell Prolif. 2020. https://doi.org/10.1111/cpr.12947.

7. Ahn JY, Sohn Y, Lee SH, Cho Y, Hyun JH, Baek YJ, et al. Use of convalescent plasma therapy in two COVID-19 patients with acute respiratory distress syndrome in Korea. J Korean Med Sci. 2020;35(14):e149-56. 
8. Duan K, Liu B, Li C, Zhang H, Yu T, Qu J, et al. Effectiveness of convalescent plasma therapy in severe COVID-19 patients. Proc Natl Acad Sci USA. 2020;117(17):9490-6.

9. Shen C, Wang Z, Zhao F, Yang Y, Li J, Yuan J, et al. Treatment of 5 critically ill patients with COVID-19 with convalescent plasma. JAMA. 2020;323(16):1582-9.

10. Zhang B, Liu S, Tan T, Huang W, Dong Y, Chen L, et al. Treatment with convalescent plasma for critically ill patients with severe acute respiratory syndrome coronavirus 2 infection. Chest. 2020;158(1):e9-13.

11. Ye M, Fu D, Ren Y, Wang F, Wang D, Zhang F, et al. Treatment with convalescent plasma for COVID-19 patients in Wuhan, China. J Med Virol. 2020;92(10):1890-901.

12. Zeng QL, Yu ZJ, Gou JJ, Li GM, Ma SH, Zhang GF, et al. Effect of convalescent plasma therapy on viral shedding and survival in patients with coronavirus disease 2019. J Infect Dis. 2020;222(1):38-43.

13. Budhiraja S, Dewan A, Aggarwal R, Singh O, Juneja D, Pathak S, et al. Effectiveness of convalescent plasma in Indian patients with COVID-19. Blood Cells Mol Dis. 2021. https://doi.org/10.1016/j.bcmd.2021.102548.

14. Li L, Zhang W, Hu Y, Tong $X$, Zheng S, Yang J, et al. Effect of convalescent plasma therapy on time to clinical improvement in patients with severe and life-threatening COVID-19: a randomized clinical trial. JAMA. 2020;324(5):460-70.

15. Joyner MJ, Wright RS, Fairweather D, Senefeld JW, Bruno KA, Klassen SA, et al. Early safety indicators of COVID-19 convalescent plasma in 5000 patients. J Clin Invest. 2020;130(9):4791-7.

16. Yang $X, Y u Y, X u$ J, Shu H, Ja Xia, Liu H, et al. Clinical course and outcomes of critically ill patients with SARS-CoV-2 pneumonia in Wuhan, China: a single-centered, retrospective, observational study. Lancet Respir Med. 2020;8(5):475-81.

17. National Health Commission of the People's Republic of China. The protocol of convalescent plasma treatment to patients with COVID-19 (version 2). 2020. http://www.gov.cn/zhengce/zhengceku/2020-03/05/5487145/ files/b1c12354cf404d629fee44738543627f.pdf. Accessed 17 May 2021.

18. World Health Organization. Clinical management of severe acute respiratory infection when novel coronavirus (nCoV) infection is suspected. 2020. https://apps.who.int/iris/handle/10665/331446? search-result= true\&query $=10665 \% 2 F 331446 \&$ scope $=\& r p p=10 \&$ sort_by $=$ score \& order=desc. Accessed 17 May 2021

19. The National Health Commission of the People's Republic of China. National recommendations for diagnosis and treatment of COVID-2019 (version 7). 2020. https://www.nhc.gov.cn/yzygj/s7653p/202003/46c92 94a7dfe4cef80dc7f5912eb1989/files/ce3e6945832a438eaae415350a8ce9 64.pdf. Accessed 17 May 2021.

20. The National Health Commission of the People's Republic of China. Prevention and control strategy of 2019-novel coronavirus (version 5). 2020 http://www.nhc.gov.cn/jkj/s3577/202002/a5d6f7b8c48c451 c87dba1488 9b30147.shtml. Accessed 17 May 2021.

21. World Health Organization. Blood donor selection: guidelines on assessing donor suitability for blood donation. 2012. https://apps.who.int/iris/ handle/10665/76724. Accessed 20 July 2021.

22. National Health Commission of the People's Republic of China. National standard operating procedures of blood collection. 2019. http://www. nhc.gov.cn/yzygj/s7658/201905/bdd4f4ccd15c4201bfb6d9e7492d7fab/ files/9c6c4c3a40a64bf786f5b5d8ee08b220.pdf. Accessed 20 July 2021.

23. National Health Commission of the People's Republic of China. National standard operating procedures of plasma collection. 2011. http://www. nhc.gov.cn/cmsresources/mohyzs/cmsrsdocument/doc12843.rar. Accessed 20 July 2021.

24. Bernheim A, Mei X, Huang M, Yang Y, Fayad ZA, Zhang N, et al. Chest CT findings in coronavirus disease-19 (COVID-19): relationship to duration of infection. Radiology. 2020. https://doi.org/10.1148/radiol.2020200463.

25. Baum A, Ajithdoss D, Copin R, Zhou A, Lanza K, Negron N, et al. REGNCOV2 antibodies prevent and treat SARS-CoV-2 infection in rhesus macaques and hamsters. Science. 2020:370(6520):1110-5.

26. Weinreich DM, Sivapalasingam S, Norton T, Ali S, Gao H, Bhore R, et al. REGN-COV2, a neutralizing antibody cocktail, in outpatients with Covid19. N Engl J Med. 2021;384(3):238-51.

27. WHO Blood Regulators Network. Position paper on use of convalescent plasma, serum or immune globulin concentrates as an element in response to an emerging virus. 2017. https://www.who.int/bloodprodu
cts/brn/2017_BRN_PositionPaper_ConvalescentPlasma.pdf?ua=1. Accessed 17 May 2021.

28. Mair-Jenkins J, Saavedra-Campos M, Baillie JK, Cleary P, Khaw FM, Lim WS, et al. The effectiveness of convalescent plasma and hyperimmune immunoglobulin for the treatment of severe acute respiratory infections of viral etiology: a systematic review and exploratory meta-analysis. J Infect Dis. 2015;211(1):80-90.

29. Xu TM, Lin B, Chen C, Liu LG, Xue Y. Non-optimal effectiveness of convalescent plasma transfusion and hydroxychloroquine in treating COVID-19: a case report. Virol J. 2020. https://doi.org/10.1186/s12985-020-01354-6.

30. Joyner MJ, Bruno KA, Klassen SA, Kunze KL, Johnson PW, Lesser ER, et al. Safety update: COVID-19 convalescent plasma in 20,000 hospitalized patients. Mayo Clin Proc. 2020;95(9):1888-97.

31. Cheng Y, Wong R, Soo YO, Wong WS, Lee CK, Ng MH, et al. Use of convalescent plasma therapy in SARS patients in Hong Kong. Eur J Clin Microbiol Infect Dis. 2005;24(1):44-6.

32. Hegerova L, Gooley TA, Sweerus KA, Maree C, Bailey N, Bailey M, et al. Use of convalescent plasma in hospitalized patients with COVID-19-case series. Blood. 2020;136(6):759-62.

33. Salazar E, Perez KK, Ashraf M, Chen J, Castillo B, Christensen PA, et al. Treatment of coronavirus disease 2019 (COVID-19) patients with convalescent plasma. Am J Pathol. 2020;190(8):1680-90.

34. Rasheed A, Fatak D, Hashim H, Maulood M, Kabah K, Almusawi Y, et al. The therapeutic potential of convalescent plasma therapy on treating critically-ill COVID-19 patients residing in respiratory care units in hospitals in Baghdad. Iraq Infez Med. 2020;28(3):357-66.

35. Salazar E, Christensen PA, Graviss EA, Nguyen DT, Castillo B, Chen J, et al. Treatment of coronavirus disease 2019 patients with convalescent plasma reveals a signal of significantly decreased mortality. Am J Pathol. 2020;190(11):2290-303.

36. Olivares-Gazca JC, Priesca-Marin JM, Ojeda-Laguna M, Garces-Eisele J, Soto-Olvera S, Palacios-Alonso A, et al. Infusion of convalescent plasma is associated with clinical improvement in critically ill patients with COVID19: a pilot study. Rev Invest Clin. 2020;72(3):159-64.

37. Erkurt MA, Sarici A, Berber I, Kuku I, Kaya E, Ozgul M. Life-saving effect of convalescent plasma treatment in covid-19 disease: clinical trial from eastern Anatolia. Transfus Apher Sci. 2020. https://doi.org/10.1016/j.trans ci.2020.102867.

38. Bandopadhyay P, Rozario RD, Lahiri A, Sarif J, Ray Y, Paul SR, et al. Nature and dimensions of the systemic hyper-inflammation and its attenuation by convalescent plasma in severe COVID-19. J Infect Dis. 2021. https:// doi.org/10.1093/infdis/jiab010/6089119.

39. Simonovich VA, Burgos Pratx LD, Scibona P, Beruto MV, Vallone MG, Vazquez C, et al. A randomized trial of convalescent plasma in Covid-19 severe pneumonia. N Engl J Med. 2020;384(7):619-29.

40. Agarwal A, Mukherjee A, Kumar G, Chatterjee P, Bhatnagar T, Malhotra P, et al. Convalescent plasma in the management of moderate covid-19 in adults in India: open label phase II multicentre randomised controlled trial (PLACID Trial). BMJ. 2020. https://doi.org/10.1136/bmj.m3939.

41. Piechotta V, Iannizzi C, Chai KL, Valk SJ, Kimber C, Dorando E, et al. Convalescent plasma or hyperimmune immunoglobulin for people with COVID-19: a living systematic review. Cochrane Database Syst Rev. 2021;5: CD013600.

42. Moore JB, June CH. Cytokine release syndrome in severe COVID-19. Science. 2020;368(6490):473-4.

43. Channappanavar R, Perlman S. Pathogenic human coronavirus infections: causes and consequences of cytokine storm and immunopathology. Semin Immunopathol. 2017;39(5):529-39.

44. Ruan Q, Yang K, Wang W, Jiang L, Song J. Clinical predictors of mortality due to COVID-19 based on an analysis of data of 150 patients from Wuhan, China. Intensive Care Med. 2020;46(5):846-8.

45. Fehr AR, Channappanavar R, Perlman S. Middle East Respiratory Syndrome (MERS): emergence of a pathogenic human coronavirus. Annu Rev Med. 2017;68:387-99.

\section{Publisher's Note}

Springer Nature remains neutral with regard to jurisdictional claims in published maps and institutional affiliations. 